\title{
A Method to Prepare TEM Specimens by Focused Ion Beam Milling for $\mathrm{Cu} /$ diamond Composites
}

\author{
Luhua Wang ${ }^{1,2}$, Hailong Zhang ${ }^{1}$, Xitao Wang ${ }^{1}$, Jinguo Wang ${ }^{2}$ and Moon J. Kim² \\ 1. State Key Laboratory for Advanced Metals and Materials, University of Science and Technology \\ Beijing, Beijing 100083, China \\ 2. Department of Materials Science and Engineering, The University of Texas at Dallas, Richardson, \\ Texas 75080, USA
}

With the advent of new technology era, electronic devices are developing towards more versatility, smaller size and higher integration, which lead to rapid increase in heat flux density. Traditional packaging materials such as $\mathrm{Cu}-\mathrm{W}, \mathrm{Cu}-\mathrm{Mo}$, Invar, Kovar, and $\mathrm{Cu} / \mathrm{SiC}$ are no longer adequate as heat spreaders and heat sinks $[1,2]$. As a promising candidate for thermal management materials, diamond particles reinforced copper matrix $(\mathrm{Cu}$ /diamond) composites have attracted a great deal of attention due to their superior thermal conductivity (TC) and tailorable coefficient of thermal expansion (CTE) [3]. It is well known that the interface between the diamond particle reinforcement and the $\mathrm{Cu}$ matrix plays a paramount role in determining the thermal properties of the composites. Thus, the characterization of the interface in the composites is of great importance.

The focused ion beam (FIB) lift-out technique has been successfully used to prepare nearly all types of transmission electron microscopy (TEM) specimens [4]. However, for $\mathrm{Cu}$ /diamond composites, the sharp difference in hardness between the diamond particle reinforcement and the $\mathrm{Cu}$ matrix makes it extremely difficult to obtain a high-quality TEM lamella. So far the delicate characterization about the interface and how the interface influences thermal properties of $\mathrm{Cu}$ /diamond composites are rarely presented in literature. Figure 1(a) shows the polished surface morphology of $\mathrm{Cu}$ /diamond composites fabricated by a gas pressure infiltration (GPI) method. It reveals that the diamond particles are uniformly distributed in the $\mathrm{Cu}$ matrix. From Figure 1(b), it can be seen that the interface between the diamond particle reinforcement and the $\mathrm{Cu}$ matrix is tilted. If we make the TEM specimen by the conventional milling from top to bottom, all the "soft" $\mathrm{Cu}$ matrix that is on the top side of the interface is easy to remove, while the "hard" diamond particle reinforcement on the bottom side still exhibits high resistance to be thinned down. The interface is thus non-existent. On the other hand, if the interface is vertical, the diamond phase and $\mathrm{Cu}$ phase can be exposed to ion beam simultaneously. Due to the huge hardness difference, the milling rate of $\mathrm{Cu}$ matrix is much faster than the diamond particle reinforcement. The two phases cannot be milled uniformly, which makes the interface between them severely damaged. It is extremely difficult to obtain a high-quality TEM foil to characterize the interface of the $\mathrm{Cu} /$ diamond composite.

What we need to do is to make the interface horizontal and then turn the diamond phase to the top side of the interface. In this way, when the sample is thinned down, the diamond phase and $\mathrm{Cu}$ phase can be milled uniformly from top to bottom and the interface is fully preserved. Taking these into account, a modified preparation method is developed as shown in Figure 2. Figure 2(c) shows the tilted interface that is observed from the trench of Figure 2(b). After first cut (Figure 2(d)), two rotations are followed. The first rotation is to rotate the sample stage to a certain angle (Figure 2(e)). When the stage rotation is done, a nanomanipulator is used to lift out the sample and the rotation of nanomanipulator with the sample is followed (Figure 2(f)). With the combination of the two rotations, the interface becomes 
horizontal and the diamond phase is located on the top side (Figure $2(\mathrm{~g})$ ). Next, the nanomanipulator is approached to the TEM grid (Figure 2(h)). When the sample is attached on the grid, Pt is used to bond the sample with the grid. After this, the nanomanipulator is cut and retracted. After thinning down, a high-quality TEM lamella with a thickness of less than $100 \mathrm{~nm}$ is achieved (Figure 2(i)). Figure 2(j) shows a STEM ABF image in which the interfacial structure of the $\mathrm{Cu}$ /diamond composite reinforced with Ti coated diamond particles is clearly presented. This method provides guidance to preparing highquality TEM lamellas of difficult-to-machine hard phase reinforced "soft" metal matrix composites.

\section{References:}

[1] C Zweben, J. Adv. Mater. 39 (2007), p. 3.

[2] T Schubert, et al., Compos. Part A: Appl. Sci. Manuf. 38 (2007), p. 2398.

[3] J W Li, et al., Scr. Mater. 109 (2015), p. 72.

[4] L A Giannuzzi, R Geurts and J Ringnalda, Microsc. Microanal. 11(suppl 2) (2005), p. 828.

[5] The authors acknowledge fundings from the International Science and Technology Cooperation Program of China (No. 2014DFA51610) and the Louis Beecherl, Jr Endowment Funds.

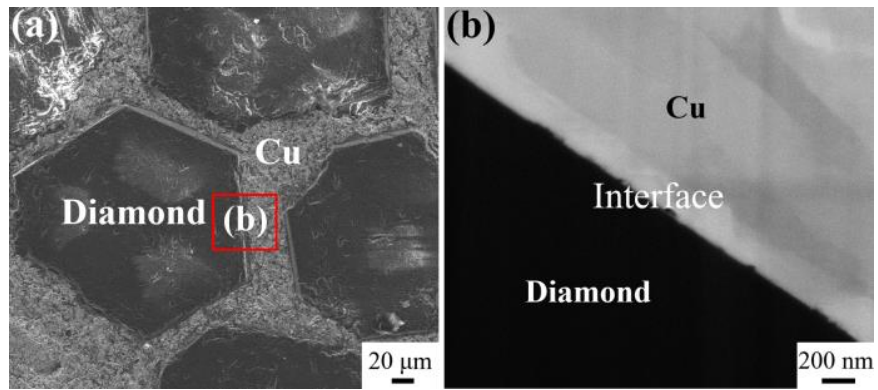

Figure 1. SEM images of (a) the polished surface and (b) the cross-sectional surface marked in (a) of the $\mathrm{Cu}$ /diamond composite.

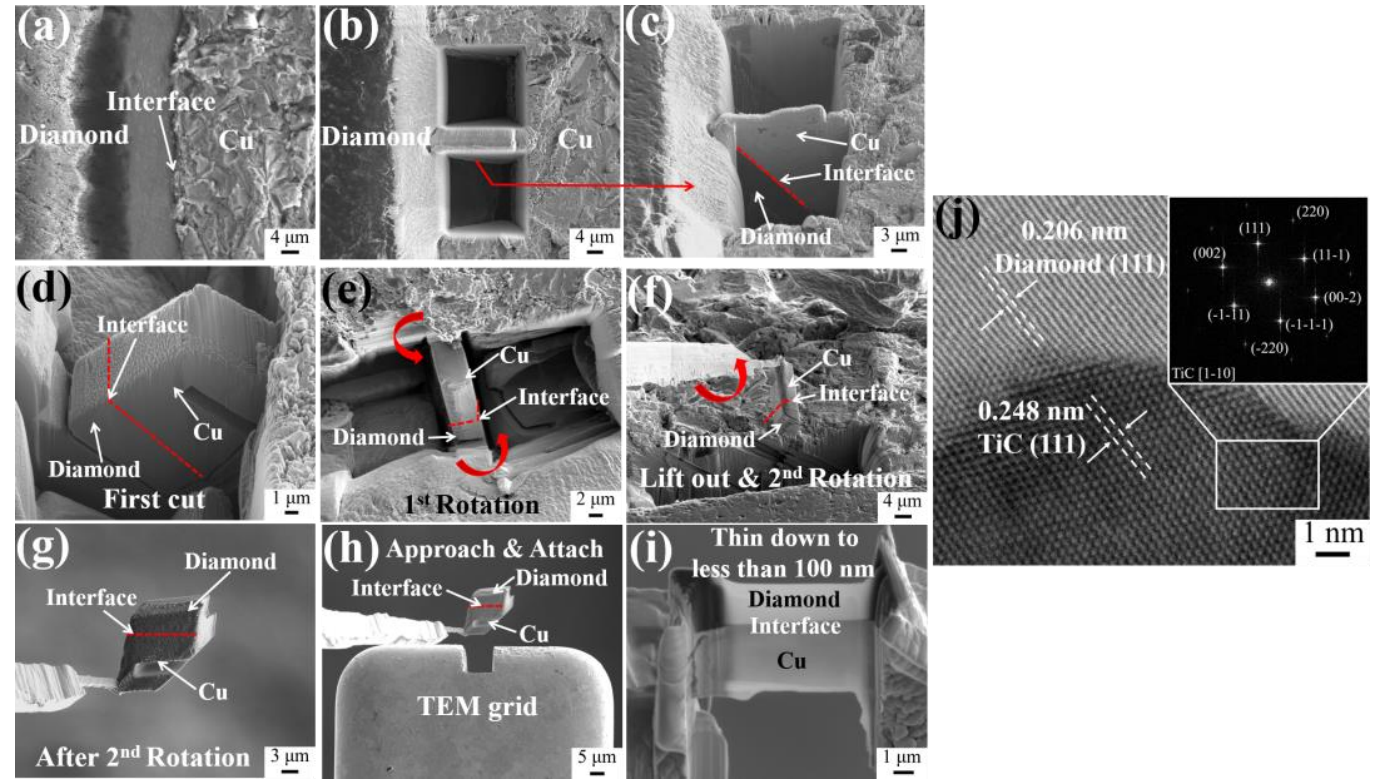

Figure 2. $(\mathrm{a}-\mathrm{i})$ SEM images showing the procedure to prepare a high-quality TEM specimen of the $\mathrm{Cu}$ /diamond composite and (j) STEM ABF image taken at the interface in the Cu/diamond composite reinforced with Ti coated diamond particles. 